\title{
Study and design of cladding power stripper for high power fiber laser systems
}

\author{
Haixia An, Xiaogang Liu, and Zhiyue Bi \\ Institute of Systems Engineering, CAEP, Mianyang 621900, China \\ (Received 19 April 2016; revised 26 June 2016; accepted 12 July 2016)
}

\begin{abstract}
Heat handling has been a significant problem of the high power fiber laser systems as the output power increases rapidly. Cladding power stripper (CPS) which is used to deal with the unwanted optical power and light is required for higher cooling ability. So the methods of stripping the unwanted light attracted much attention recently, and the thermal effect is given. However, few investigations focus on the dissipation of the heat converted from the unwanted light. In this paper, an approach of active cooling for CPS is demonstrated. This is achieved by using microchannel cooling technology in heat sinking in CPS to improve the efficiency of heat exchange. In order to explain the mechanism of CPS the function of it and consistence of categories of the unwanted light are detailed firstly. Then microchannel heat sinking is proposed and verified by the heat exchange theory. At last, the design of the CPS with microchannel heat sinking is shown and following experiment is conducted. The final temperature of the device with $1000 \mathrm{~W}$ cladding power was demonstrated at last to verify the ability of heat distribution of the CPS component. This suggests that these CPSs can be used to stripe a thousand of watts of light in high power double cladding fiber lasers.
\end{abstract}

Keywords: cladding power stripper; double cladding fiber; high power laser system; microchannel

\section{Introduction}

Fiber laser systems have many advantages over other laser systems, including high beam quality, high conversion efficiency, less-cost, light weight and small volume ${ }^{[1]}$. They have been widely applied in areas, such as optical sensing, advanced industrial processing, medical industries and military defense ${ }^{[2]}$. With the development of the double cladding fiber technology and laser diode pump technology, output power of the fiber laser systems has been increased rapidly. On the other hand, the unwanted light and optical power increases. Thermal management has been a significant problem of the high power fiber laser systems as the output power increases rapidly. It is critical to manage and remove the wasted heat to protect the fiber and other components of the system. So methods of stripping the unwanted light in high power fiber laser attracted much attention recently and the thermal effect is given. However, few investigations focus on the dissipation of the heat converted from the unwanted light. In this paper, a cooling method of using microchannel in cladding power stripper (CPS) was proposed and the final temperature of the CPS is given by a thermal camera.

Correspondence to: H. An, Institute of Systems Engineering, CAEP, Mianyang 621900, China. Email: 409anhx@caep.cn

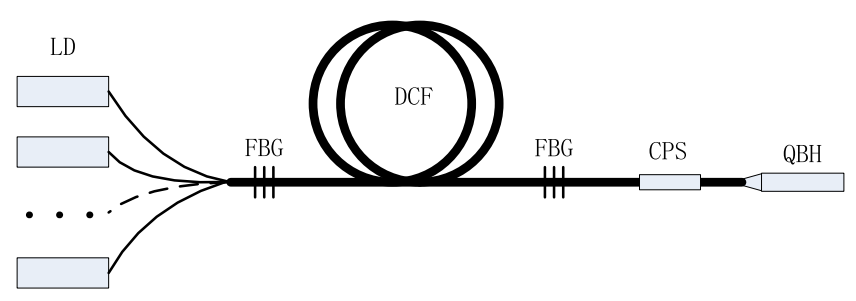

Figure 1. Configuration of DCFLS.

\section{Function of cladding power stripper}

The configuration of a typical double cladding fiber laser system (DCFLS) is schematically shown in Figure 1. The fiber was cladding-pumped by high power diode stacks and then propagate and amplify the pump light. High reflector and output coupler (low reflector) fiber Bragg gratings (FBGs) are spliced or etched on the fiber in the system. It is perfect for a system that all signal light transmit in the core and inner cladding and all pumping light is absorbed by the core. However, there is still unwanted light exit in reality. CPS is a component of the system used to remove this unwanted light and distribute the heat converted by it. Quartz Block for high power laser can help get collimated light beam and avoid thermal damage at the end of fiber. 
The unwanted light propagating in the cladding mainly consists of three parts: amplified spontaneous emission (ASE), residual pump light at the end of the gain fiber and core light leaking into the cladding or being reflected into cladding $^{[3]}$. Before the output the unwanted light has to be removed for it would use free space optics of a complicated laser system. The unwanted light convert to heat and this may heat up the optics so that the core light cannot focus tightly. Furthermore, the heat converted by the unwanted light may burn the components of the system or deteriorate the function when several systems are combined.

In DCFLS the amount of unwanted optical power and light must be dealt with so that the output light has high beam quality. Many researches have been done to stripper the unwanted light from the cladding. Removing the original fluoroacrylate jacket and recoating the exposed fiber with a high index polymer coating can strip over more than hundred watts of residual light ${ }^{[3]}$. Fabricated using an etching technique successfully tested that it has good behavior in stability and thermal so it can be used for higher power laser systems $^{[4]}$. Corroding the inner cladding layer into special shape can strip the power of cladding light uniformly over the length of the fiber rather than other previous methods ${ }^{[5]}$. By using a glass capillary collapsed onto the fiber to match the index cladding of the fiber heat to be dissipated over a much larger surface area and for a more reliable device ${ }^{[6]}$. These researches removed the unwanted light successfully and the critical work after converting the unwanted light to heat is how to distribute and cool the CPS. Some of the researches give the cooling method of their test, including air cooling and cooling water based plate. But as the output power of DCFLS increases, the traditional cooling method could not suffer the strong heat, so we propose the microchannel heat sink of CPS.

\section{Microchannel heat sink}

Microchannel heat sink is one of the most promising high efficiency heat exchange technologies and it has been used in high power solid-state lasers ${ }^{[7]}$. The work process of microchannel heat sink is schematically shown in Figure 2. The red arrows at the bottom are heat radiated from the fiber in the tube, which was stripped of the outer cladding to radiate unwanted power utmost.

According to the heat exchange theory, the heat dissipated can be expressed in the equation:

$$
Q=h A\left(t_{i}-t_{l}\right)
$$

where $Q$ is the heat dissipated by cooling device, CPS in this paper; $h$ is convective heat transfer coefficient of cooling liquid; $A$ is the area of microchannel surface; $t_{i}$ is temperature of the inner face of the microchannel; $t_{l}$ is temperature of cooling liquid.

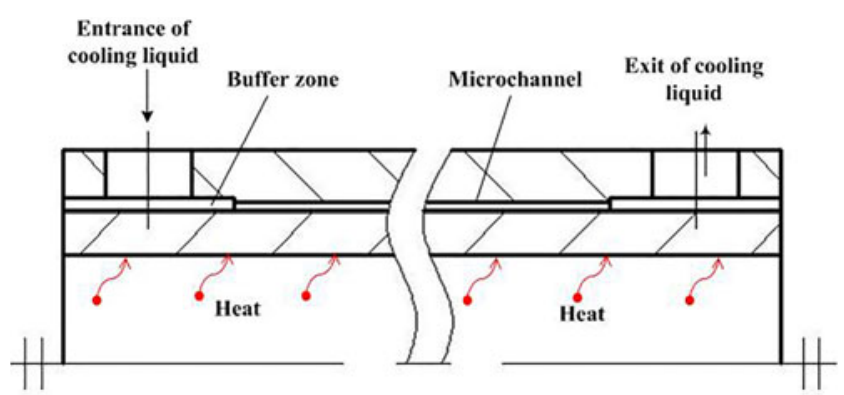

Figure 2. Schematic diagram of microchannel heat sink.

The temperature of the inner face of the microchannel depends on heat converted from unwanted light. In order to improve the heat dissipated there are three methods: improve the convective heat transfer coefficient $h$, broaden the area of microchannel surface $A$, reduce the temperature of cooling liquid.

The dimension of the microchannel is very small so the cooling liquid is considered as laminar flow and the equation below can express the state.

$$
N_{u}=\frac{h \cdot D_{h}}{k}=\mathrm{const},
$$

where $N_{u}$ is Nusselt number, it can be considered as a constant in laminar flow state; $h$ is convective heat transfer coefficient of cooling liquid as has introduced; $D_{h}$ is the diameter of the cooling liquid flow; $k$ is thermal conductivity of cooling liquid.

It is easy to get this:

$$
h=\frac{N_{u} \cdot k}{D_{h}} .
$$

The convective heat transfer coefficient depends on thermal conductivity and diameter of cooling liquid. For microchannel the diameter is $0.1-1 \mathrm{~mm}^{[8]}$, the convective heat transfer coefficient can be improved significantly. Replacing the convective heat transfer coefficient in Equation (1), the heat dissipated increases greatly. The research also verified that the average temperature and the peak temperature trend to a considerable reduction and a better temperature distribution when the channel diameter decreases ${ }^{[9]}$. In a word, microchannel is an efficient cooling method that can be used in CPS.

\section{Design and experiment of CPS}

The microchannel cooling technology is used when designing the CPS structure. We choose the rectangular header shapes for the microchannel for it provides better flow velocity uniformity than the trapezoidal and triangular headers. The structure of the CPS consists of four parts: heat 


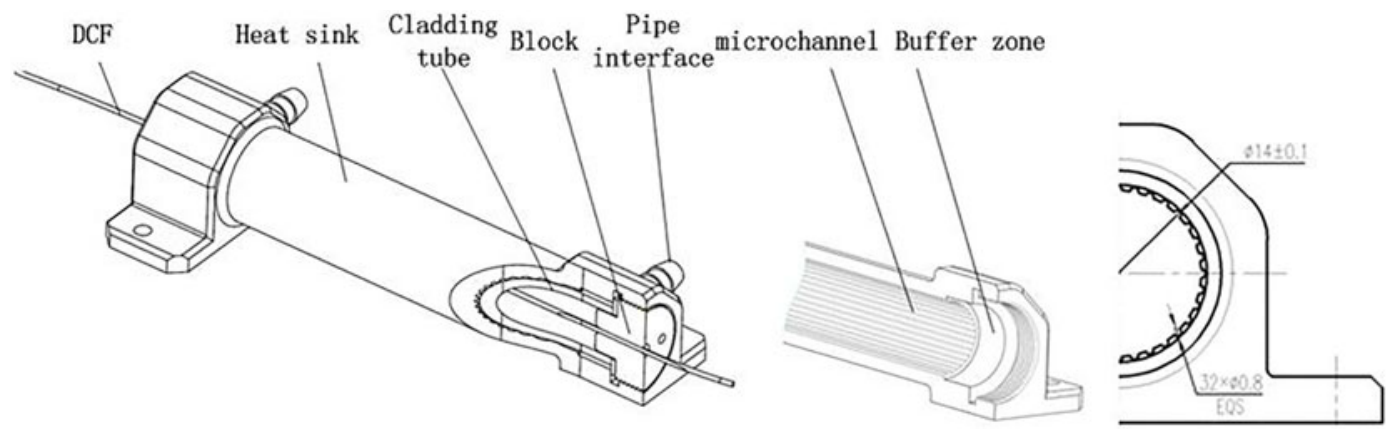

Figure 3. Design of CPS.

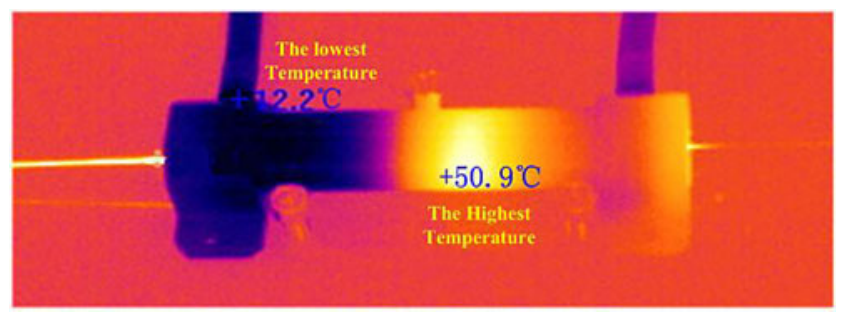

Figure 4. Thermal effect photo of $1000 \mathrm{~W}$ cladding power.

sink, cladding tube, block and pipe interface. The heat sink is made of aluminum alloy, which is easy to process. The structure of the CPS is shown in Figure 3.

The CPS is used to take off the heat converted from the unwanted light. In the center of the CPS is the double cladding fiber surrounded by a tube. The cladding tube let the unwanted light disperse on the heat sinking and the unwanted light convert to heat when contacting the inner surface of the heat sinking. The fuscous coat can absorb the heat and dissipate it then with the flow of the cooling liquid. The conventional rectangular microchannel can be seen clearly in the figure. The cooling liquid flows in the rectangular microchannel to take the heat off quickly as has been explained in part 2. The part of block is used to encapsulate the cladding tube and the heat sinking and the pagoda interface provide the way in and out for the cooling liquid.

The experiment of the CPS is conducted under the light propagate in fiber is $10 \mathrm{~kW}$ and the cladding power is $1000 \mathrm{~W}$, which means that the unwanted light consists about $10 \%$ of the fiber laser power. Water is used as the cooling liquid and the diameter of the entrance pipeline is $6 \mathrm{~mm}$ and the hydraulic flux is $1 \mathrm{~L} \mathrm{~min}{ }^{-1}$. At the end of the experiment the temperature of the device is recorded by a thermal camera. The photo is shown in Figure 4.
The highest working temperature of a CPS is no higher than $60^{\circ} \mathrm{C}$ for there is colloidal macromolecule substances in fiber. The peak temperature of the CPS is $50.9^{\circ} \mathrm{C}$. It is lower than $60^{\circ} \mathrm{C}$. So the CPS with microchannel heat sinking is sufficient for $10 \mathrm{~kW}$ DCFLSs.

\section{Conclusion}

In order to explain the mechanism of the CPS we began with introducing laser systems and enumerating the categories of unwanted light in the chain of the components. Then the microchannel heat sinking is presented and verified. On the basis of the microchannel cooling technology we show the design of the CPS. The experiment results presented in the last suggests that the CPS with microchannel heat sinking is sufficient for $10 \mathrm{~kW}$ DCFLSs. Further work needs to be done in order to optimize the structure of the CPS to meet the high cladding power and also for CPS itself.

\section{References}

1. W. Lu, W. Dong, H. Yang, P. Xu, and Z. Tang, Laser Infrared 8, 845 (2013).

2. S. Katayama and Y. Kawahito, Proc. SPIE 7195, 71951R (2009).

3. A. Wetter, M. Faucher, and B. Sévigny, Proc. SPIE 6873, 687327 (2008).

4. A. Kliner, K. Hou, M. Plötner, Ch. Hupel, Th. Stelzner, T. Schreiber, R. Eberhardt, and A. Tünnermann, Proc. SPIE 8616, $86160 \mathrm{~N}$ (2013).

5. W. Wang, J. Leng, J. Cao, S. Guo, and W. Xu, Opt. Commun. 287, 187 (2013).

6. L. Bansal, V. R. Supradeepa, T. Kremp, and C. Headley, Proc. SPIE 9344, 93440F (2015).

7. H. Zhe, OME Inf. 11, 1 (2004).

8. C. Tian, H. Xu, H. Cao, and C. Si, Chin. J. Lasers 36, 1686 (2009).

9. V. Manoj Siva, A. Pattamatta, and S. K. Das, Intl J. Heat Mass Transf. 73, 424 (2014). 